The Psychological Record, 2008, 58, 71-90

\title{
A TRANSFORMATION OF FUNCTIONS IN ACCORDANCE WITH THE NONARBITRARY RELATIONAL PROPERTIES OF SEXUAL STIMULI
}

\author{
Bryan Roche \\ National University of Ireland, Maynooth \\ Simon Dymond \\ Swansea University
}

\begin{abstract}
In two experiments, researchers examined the transformation of the nonarbitrary properties of sexual stimuli in accordance with multiple stimulus relations. First, simple discrimination and free-operant procedures were used to present small, medium, or large sexually explicit images. Contextual functions of "More than" and "Less than" were then established for two cues by using a relational pretraining procedure. Next, a linear-series conditional discrimination training design was used to train and test for the formation of three, three-member equivalence relations. Then, emergent relations of More than and Less than were tested among the stimuli. The nonarbitrary relations of More than and Less than obtaining among the sexual stimuli were arbitrarily applied to all equivalence relations and the discriminative stimuli. These findings may have implications for contemporary behavioral sex research in demonstrating that stimuli may acquire sexual and relational functions through their participation in complex relational networks.
\end{abstract}

Research on derived stimulus relations has shown that if a verbally able human being is trained, in a matching-to-sample context, to match $\mathrm{A}$ to $\mathrm{B}$ and $\mathrm{B}$ to $\mathrm{C}$, he or she will also likely match $\mathrm{B}$ to $\mathrm{A}$ and $\mathrm{C}$ to $\mathrm{B}$ (symmetry), A to $\mathrm{C}$ (transitivity), and $\mathrm{C}$ to $\mathrm{A}$ (equivalence) without reinforcement (see Fields, Adams, Verhave, \& Newman, 1990; Sidman, 1986; Hayes, BarnesHolmes, \& Roche, 2001). Another feature of derived relations that is of particular importance in the current context is the derived transformation of functions. Specifically, if one stimulus in a derived relation is established as a conditioned sexual stimulus, for instance, the functions of other stimuli

Data from this article were presented at the Behaviour Analysis in Ireland meeting, Maynooth, Co. Kildare, May 2002, and at the annual convention of the Association for Behavior Analysis, San Francisco, May 2003.

We thank Rob Whelan for his comments on an earlier draft.

Address correspondence to Bryan Roche, Department of Psychology, National University of Ireland, Maynooth, Co. Kildare, Ireland. Email: Bryan.T.Roche@nuim.ie 
in the equivalence relation are transformed accordingly (Dymond \& Rehfeldt, 2000). That is, if the C1 stimulus in the foregoing example is associated explicitly with a sexually arousing visual image, then A1 but not A2 will also acquire sexual arousal-eliciting functions (see Roche \& Barnes, 1997).

To date, a wide variety of stimulus function transformations has been demonstrated in accordance with equivalence relations (e.g., Barnes \& Keenan, 1993; Dougher, Augustson, Markham, Greenway, \& Wulfert, 1994; Dougher, Perkins, Greenway, Koons, \& Chiasson, 2002; Dymond \& Barnes, 1995; Rehfeldt \& Hayes, 1998; Smeets \& Barnes-Holmes, 2003; see Dymond \& Rehfeldt, 2000, for a review) and derived relations other than equivalence, such as Sameness, Opposition, and Difference (Dymond \& Barnes, 1996; Steele \& Hayes, 1991; Roche \& Barnes, 1996, 1997; Whelan \& Barnes-Holmes, 2004), More than and Less than (Dymond \& Barnes, 1995; O’Hora, Roche, Barnes-Holmes, \& Smeets, 2002; Whelan, Barnes-Holmes, \& Dymond, 2006), and Before and After (Barnes-Holmes, Hayes, Dymond, \& O’Hora, 2001; O’Hora, Barnes-Holmes, Roche, \& Smeets, 2004). For example, Roche and Barnes (1997) exposed participants to a relational pretraining procedure to establish contextual functions of Same and Opposite for two arbitrary stimuli. Specifically, across several training trials participants were presented with sample stimuli and three comparison stimuli that were related to each other along a physical dimension. For example, one set of comparison stimuli consisted of a long line, a medium line, and a short line. When a participant was given a shortline sample stimulus in the presence of the Opposite contextual cue, choosing the long-line comparison stimulus was reinforced. However, given the Same contextual cue and a short line sample, choice of the short-line comparison was reinforced. Given relational pretraining across a sufficient number of such exemplars (e.g., circles, stars, rectangles), contextual control by the arbitrary cues was established and was evident across novel sets of samples and comparisons. In a subsequent training stage, a series of arbitrary stimulus relations was trained in the presence of the contextual cues, and a series of derived stimulus relations emerged during testing. The trained relations were Same/A1-B1, Same/A1-C1, Opposite/A1-B1, and Opposite/A1-C1, and the derived stimulus relations were Same/B1-C1, Same/B2-C2, Opposite/B1-C2, and Opposite/B2-C1.

After successful performance on the arbitrary relations test, participants were then exposed to a differential conditioning procedure during which sexual arousal functions were established for the B1 stimulus only by pairing it with contiguous presentations of sexually explicit film clips and by pairing B2 with the absence of sexually explicit material. During the critical probes for a transformation of function, participants were repeatedly presented with the $\mathrm{C} 1$ and $\mathrm{C} 2$ stimuli but in the absence of film clips. Participants showed a transformation of the functions of the $C$ stimuli in accordance with the relational network and the established respondent functions of the B1 stimulus. More specifically, electrodermal sexual arousal functions (measured as skin resistance responses) emerged for $\mathrm{Cl}$ (i.e., participants derived that the conditioned sexual stimulus $\mathrm{B} 1$ is the same as $\mathrm{C} 1$ ) but nonsexual functions of $\mathrm{B} 2$ emerged for $\mathrm{C} 2$ when $\mathrm{C} 2$ was presented (i.e., the participants remained relaxed). A further study by Roche, Barnes-Holmes, Smeets, Barnes-Holmes, \& McGeady (2000) replicated and extended these findings by bringing the derived transformation effect under further contextual control.

Together these findings indicate that the study of derived stimulus 
relations provides an opportunity to examine parameters of complex human sexual behavior that are not easily captured within a traditional respondent or operant paradigm. In particular, the transformation of functions in accordance with derived relations suggests that sexual arousal in the world outside the laboratory may sometimes arise in the absence of direct reinforcement or respondent conditioning (Roche \& Barnes, 1998). However, research to date has examined only the transformation of experimentally established respondent or operant response functions in accordance with arbitrary derived relations. What is missing from the literature is an analysis of the complex derived transformation of functions by the nonarbitrary and naturally occurring relational properties of stimuli, such as primary reinforcers. More specifically, in a naturalistic setting it is likely that verbally able humans respond to the nonarbitrary relationship between various stimuli in arbitrary terms (Hayes, 1994). For instance, a verbally able adult not only can select between two stimuli discriminative for different amounts of sexual reinforcement but also can discriminate the relation between these reinforcers. It is in this sense that relational frame theory (RFT; Hayes et al., 2001) refers to stimulus relations as arbitrarily applicable. That is, verbally able individuals can tact the specific types of relations that obtain between an infinite variety of events.

From the RFT perspective, the formal properties of stimuli participate in derived relations. Thus the formal relational features of stimuli (e.g., size) themselves become abstractions that can be applied under contextual control to any set of relata. Most RFT research to date has focused only on the truly arbitrary nature of relational frames. What is required, however, is an analysis of the role of formal properties in the contextual control of relational responding and the effect the participation of formal features in relational networks has on the transformation of function.

The discrimination of relations between primary reinforcers, such as food and sexual stimuli, likely leads to all sorts of complex transformations of functions among related events. The interesting point is that the emergent relations need not be of a kind that has been trained formally at any stage, as is typically the case in experimental preparations. For instance, imagine an individual for whom two stimuli differ in their potency as sexual reinforcers. Now further imagine that these stimuli participate in separate verbal relations (e.g., equivalence relations) with a range of other stimuli, such as words and olfactory or tactile stimuli. It is likely that a verbally able human will respond to the members of these different verbal relations in comparative terms, despite comparative relations having never been formally established between the sexual stimuli or any of the other relata. In effect, the derivation of relations of more than and less than between the relata occurs because of the derived transformation of functions in accordance with arbitrary relations by the formal properties of the sexual reinforcers. If such a process can be demonstrated experimentally, it would further illustrate the ubiquity and spontaneity of the derived transformation-of-functions effect. It would also represent an empirical analysis of the interaction between the formal and arbitrary relational features of stimuli and how these features conspire to produce unique transformation-of-function effects.

Previous research has demonstrated the transformation of both selfdiscriminative and consequential functions but not sexual stimulus functions, in accordance with multiple stimulus relations of More than and 
Less than (Dymond \& Barnes, 1995; Whelan et al., 2006), and no study to date has examined the complex transformation of functions by the nonarbitrary properties of formally related stimuli. With the present study we sought to extend the analysis of the transformation of stimulus functions in accordance with complex networks of More than and Less than relations. Across two experiments, we examined a transformation in the relational properties of a range of arbitrary stimuli by virtue of their participation in derived relations with sexual stimuli of varying size. In Experiment 1, participants were exposed to a simple discrimination procedure during which small, medium, or large sexually explicit pictures were presented after an appropriate keypressing response to one of three discriminative stimuli. In Experiment 2, participants were exposed to a stimulus pairing procedure followed by a freeoperant baseline phase in which participants' preferences for the differentsized pictures were assessed. In both experiments contextual functions of More than and Less than were then established for two arbitrary cues. Finally three, three-member equivalence relations were trained and tested, where the A stimuli were the small, medium, and large sexually explicit pictures and the $\mathrm{B}$ and $\mathrm{C}$ stimuli were nonsense syllables. A relational test phase then probed for emergent More-than and Less-than relations between the $B$ and $\mathrm{C}$ stimuli and between the discriminative stimuli used in training. It was predicted that the nonarbitrary relations of More than and Less than obtaining among the various sexual stimuli would spontaneously emerge between the corresponding $\mathrm{C}$ stimuli from the equivalence relations.

\section{General Method}

\section{Participants}

Nine participants, eight male and one female, aged between 21 years and 35 years, were recruited from personal contacts. Three participants, two male and one female, participated in Experiment 1, and six participants, all male, participated in Experiment 2. All participants provided informed consent that they were over eighteen years of age and were aware that they would be exposed to sexually explicit stimuli.

\section{Apparatus and Stimuli}

Participants completed all experimental phases in a small, soundattenuated room containing an Apple Macintosh iMac computer running PsyScope software (see Cohen, MacWhinney, Flatt, \& Provost, 1993; Roche, Stewart, \& Barnes-Holmes, 1999) that controlled stimulus presentations and recorded responses. The computer screen size was optimized for $800 \times 600$ pixels.

Two stimuli each consisting of six characters (!!!!!! and ??????) were used as contextual cues for More than and Less than, respectively. Stimuli used in the experiment consisted of nine three-letter nonsense syllables (e.g., CUG, JOM, ZID, PAF, MEL, LEB, VEP, QUV, ROG) arbitrarily assigned as samples and comparisons and were presented in black Times 24-point font on a white background. For the sake of clarity, the nonsense syllables are assigned alphanumeric labels here (i.e., A1, B1, C1, A2, B2, C2, A3, B3, C3), although participants never saw these labels. Sexually explicit stimuli consisted of scanned photographs of solo nude women and were obtained from several 
publications widely available throughout the United Kingdom. The pixel sizes of the small, medium, and large sexually explicit stimuli were $86 \times 112,90 \times$ 192, and $328 \times 390$, respectively.

\section{General Procedure}

There were six experimental phases in Experiment 1 and seven in Experiment 2. In Experiment 1, Phase 1, participants were trained to conditionally relate one of three colored circles with sexually explicit stimuli of differing sizes. In Phase 2, participants were exposed to nonarbitrary training and testing designed to establish contextual functions of More than and Less than. In Phase 3, A-B and B-C conditional discriminations were trained, and in Phase 4, transitivity (A-C) and equivalence (C-A) relations were tested. Phase 5 tested for emergent relations of More-than and Lessthan obtaining between the colored circles and the B and C stimuli. Finally, Phase 6 was identical to Phase 1 except that no sexually explicit stimuli were presented (i.e., extinction).

Participants were trained and tested usually in one session, ranging in length between $1 \mathrm{hr}$ and $3 \mathrm{hr}$. Where a break of a day or more occurred between sessions, participants were reexposed to the preceding phase.

\section{Experiment 1}

\section{Phase 1: Discrimination Training}

The aim of this phase was to train participants to conditionally relate each of the three colored circles to sexually explicit stimuli of differing sizes (see Figure 1). At the start of this phase, participants were presented with the following on-screen instructions:

Your task is to look at the image presented in the centre of this computer screen and to press one of the three marked buttons on the computer keyboard. You will get feedback on your choice. During this phase you will also see several sexually explicit images. If you have any questions, please ask the experimenter now. Press any key to begin.

The phase began when the participant pressed any key. One colored circle (green, blue, or red) then appeared in the center of the screen along with the words "press one of the marked keys." The participants' task was to press one of the marked keys (X, V, and $\mathrm{M}$ on the keyboard) in the presence of a particular colored circle. Responses deemed correct resulted in feedback and the presentation of a large, medium-sized, or small sexually explicit stimulus in the center of the computer screen. A different sexually explicit stimulus of the appropriate size was randomly presented for every correct trial.

Pressing the "X" key in the presence of the Green circle was followed by the word "Correct" accompanied by a brief beep and then, 2 s later, by the presentation of a large-sized sexually explicit stimulus that remained on screen for $7 \mathrm{~s}$. Pressing the "V" key in the presence of the Blue circle was followed by the word "Correct" accompanied by a brief beep and then, $2 \mathrm{~s}$ later, by the presentation of a medium-sized sexually explicit stimulus that remained on screen for $7 \mathrm{~s}$. Pressing the " $\mathrm{M}$ " key in the presence of the Red 
circle was followed by the word "Correct" accompanied by a brief ascending beep and then, $2 \mathrm{~s}$ later, by the presentation of a small-sized sexually explicit stimulus that remained on screen for $7 \mathrm{~s}$. Incorrect responses were followed by the word "Wrong" accompanied by a brief descending beep and a 2-s intertrial interval before the commencement of the next trial.

Phase 1: Discrimination Training

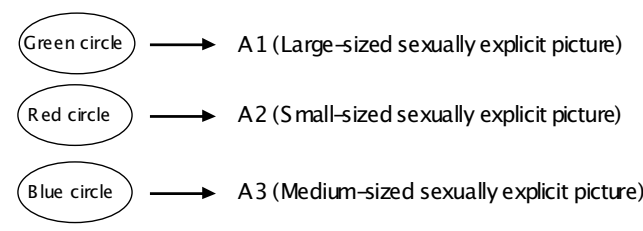

Phase 2: Nonarbitrary More Than/Less Than Pretraining and Testing

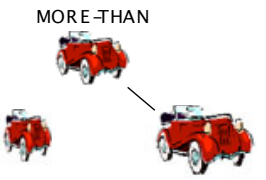

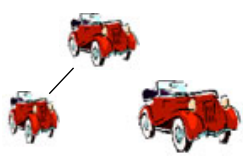

Phase 3: A-B \& B-C Relational Training

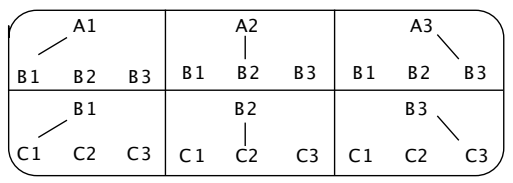

Phase 4: A-C \& C-A Relational Testing

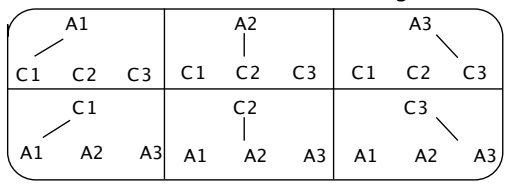

Phase 5: Probes for Combined Emergent Relations of More-Than \& Less Than and Equivalence

\begin{tabular}{|c|c|c|c|}
\hline $\int_{\mathrm{C} 3}^{\mathrm{C} 2} \mathrm{~B}_{2}^{\text {MORE-THAN }}$ & $\int_{\mathrm{C} 1}^{\mathrm{C} 2} \mathrm{~B} 2$ & $\overbrace{\mathrm{C} 1}^{\mathrm{C} 3} \mathrm{~B}_{3}$ & $\sum_{\mathrm{C} 1}^{\mathrm{C} 3} \mathrm{C2}$ \\
\hline $\begin{array}{c}\text { LESS -THAN } \\
\text { C1 }\end{array}$ & $\begin{array}{l}\text { LESS -THAN } \\
\text { C1 }\end{array}$ & $\begin{array}{c}\text { LESS -THAN } \\
\text { C } 3\end{array}$ & $\begin{array}{l}\text { LESS -THAN } \\
\text { C } 3\end{array}$ \\
\hline B 1 & C2 & C2́ & $\mathrm{C} 1$ \\
\hline
\end{tabular}

\begin{tabular}{|c|c|c|c|}
\hline $\begin{array}{c}\text { MOR E-THAN } \\
\begin{array}{c}\text { Red } \\
\text { circle }\end{array} \\
\text { C3 } 32\end{array}$ & 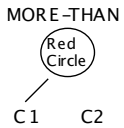 & 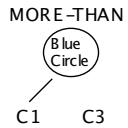 & $\begin{array}{c}\text { MORE-THAN } \\
\text { clue } \\
\text { circle } \\
\mathrm{C} 1 \\
\mathrm{C} 2 \\
\mathrm{C}\end{array}$ \\
\hline $\begin{array}{c}\text { LESS -THAN } \\
\text { (creen } \\
\text { circle }\end{array}$ & $\begin{array}{c}\text { LESS -THAN } \\
\text { Green } \\
\text { Circle }\end{array}$ & $\begin{array}{c}\text { LESS -THAN } \\
\text { (3lue } \\
\text { circle }\end{array}$ & $\begin{array}{c}\text { LESS -THAN } \\
\text { (Blue } \\
\text { Circle }\end{array}$ \\
\hline $\mathrm{C} 2$ & $\mathrm{C} 1$ & C3 & C1 \\
\hline
\end{tabular}

Figure 1. A schematic representation of Phases 1-5 in Experiment 1. Phases 2, 3, 4, and 5 were identical in Experiment 2. Solid lines indicate trained/tested relations. See text for further details.

Participants were presented with a total of 45 trials, 15 trials of each of the colored circles, which were presented quasi-randomly with no more than two consecutive trials of the same type. The criterion for completion of Phase 1 was a minimum of 42 out of 45 correct responses, and participants were immediately reexposed to this phase if the criterion was not met.

\section{Phase 2: Nonarbitrary More-Than and Less-Than Pretraining and Testing}

The purpose of this phase was to establish functions of More than and Less than for two contextual cues. Participants were presented with the following on-screen instructions:

During this phase your task is to look at the image presented at the top of this computer screen, then look at the image in the middle of the screen and finally look at the images at the bottom of the screen. You should then choose one of the images at the bottom of the screen by "clicking" on them with the mouse button. You will be given feedback on your choices and you should try to 
get as many choices correct as possible. If you have any questions please ask the experimenter now. Press any key to begin.

Participants received training with four stimulus sets (i.e., cars, footballs, bottles, and cats) in the nonarbitrary relational training tasks and four novel stimulus sets (i.e., cherries, chairs, circles, and clocks) in the nonarbitrary relational testing tasks. On all trials, presentation of a contextual cue (!!!!!! or ??????) at the top of the screen was followed by the simultaneous presentation of a sample in the center of the top half of the screen and two comparisons at the foot of the screen (see Figure 1). The stimuli remained on the screen until the participant selected one of the comparison stimuli (by clicking over it with the mouse). The position of the comparison stimuli (left or right) was counterbalanced across trials. During the nonarbitrary relational training phase, feedback was presented in the center of the screen for $1.5 \mathrm{~s}$ and consisted of the word "Correct" or the word "Wrong." All trials were followed by an intertrial interval of $2.5 \mathrm{~s}$.

In the presence of the More-than contextual cue, with a medium-sized football as the sample and smaller-sized and larger-sized footballs as the comparisons, selecting the comparison that portrayed the greater quantity of a particular object was followed by "Correct" (see Figure 1). In the presence of the Less-than contextual cue, selecting the image that portrayed the lesser quantity of a particular object was followed by "Correct" (see Figure 1). All other responses resulted in the word "Wrong."

Participants were presented with 32 trial types from the four stimulus sets once across each block of 32 trials in a random order. The criterion for completion of the nonarbitrary relational training and testing phases was a minimum of 30 out of 32 correct responses. Participants were immediately reexposed to the training tasks, with novel stimulus sets, if they failed to meet the criterion during the testing tasks and were subsequently retested, again with novel stimulus sets.

\section{Phase 3: A-B and B-C Relational Training}

The aim of this phase was to train conditional discriminations that involved the sexually explicit stimuli of differing sizes (A1, A2, A3) and nonsense syllables (B1, B2, B3, C1, C2, C3) by using a linear-series design (see Figure 1). Participants were first presented with the following instructions:

During this phase your task is to look at the image presented in the middle of the screen and then look at the images at the bottom of the screen. You should then choose one of the images at the bottom of the screen by "clicking" on them with the mouse button. You will be given feedback on your choices and you should try to get as many choices correct as possible. During this phase you will also see several sexually explicit images. If you have any questions please ask the experimenter now. Press any key to begin.

On each trial, the sample was presented in the top half of the screen, followed $1 \mathrm{~s}$ later by two comparison stimuli at the foot of the screen. Stimuli remained on the screen until the participant selected one of the comparisons, and the positions (left or right) were counterbalanced across trials.

Participants were first trained on the three A-B tasks (A1-B1, A2-B2, A3-B3). Each of these tasks was presented 6 times in a quasi-random order (i.e., once 
every three trials) for a total of 18 trials. The A stimuli were sexually explicit pictures in one of three sizes (small, medium, or large); A1 consisted of large-sized pictures, A2 consisted of small-sized pictures, and A3 consisted of medium-sized pictures. Across trials, participants were presented with multiple exemplars of large-, small-, and medium-sized pictures. When A1 (large-sized pictures) was the sample, selecting the nonsense syllable designated B1 was deemed correct. When A2 (small-sized pictures) was the sample, selecting B2 was correct. When A3 (medium-sized pictures) was the sample, selecting B3 was correct (Figure 1).

Three B-C tasks (B1-C1, B2-C2, B3-C3) were then each presented 6 times in a quasi-random order (i.e., once every three trials) for a total of 18 trials. The $\mathrm{B}$ and $\mathrm{C}$ stimuli consisted of nonsense syllables and were presented in the same manner as described above. When $\mathrm{B} 1$ was the sample, selecting $\mathrm{C} 1$ was deemed correct. When A2 was the sample, selecting C2 was correct. When A3 was the sample, selecting C3 was correct (Figure 1).

Next, a mixed block of A-B and B-C tasks was presented, with each task appearing 4 times in a block of 24 trials. In total, 60 trials were presented in this phase: $18 \mathrm{~A}-\mathrm{B}, 18 \mathrm{~B}-\mathrm{C}$, and 24 mixed A-B and B-C trials. The mastery criterion was a minimum of 9 out of 10 correct responses on each of the six tasks (A1-B1, A2-B2, A3-B3, B1-C1, B2-C2, B3-C3).

\section{Phase 4: A-C (Transitivity) and C-A (Equivalence) Relational Testing}

The aim of this phase was to test for emergent relations of transitivity (A-C) and equivalence (C-A; see Figure 1). Each task (A1-C1, A2-C2, A3-C3, C1-A1, C2-A2, C3-A3) was presented randomly 10 times for a total of 60 trials. No feedback was provided after any trial, and the mastery criterion was a minimum of 9 out of 10 correct responses on each of the six tasks. If participants did not meet this criterion, they were retrained (Phase 3) and retested (Phase 4) a maximum of four times.

\section{Phase 5: Probes for Combined Emergent Relations of More Than, Less Than, and Equivalence}

The aim of this phase was to test for derived relations of More than and Less than among the $B$ and $C$ stimuli and between the colored-shape discriminative stimuli and the $C$ stimuli (see Figure 1). Participants were presented with the same instructions used in Phase 2.

On all trials, a contextual cue (!!!!! or ??????) was presented at the top of the screen, followed by the simultaneous presentation of a sample in the center, top half of the screen and two comparisons at the foot of the screen. No feedback was delivered after any trial. Participants were presented with a total of 16 tasks, each presented 5 times for a total of 80 trials.

Half of the trials (40) tested for emergent relations among the $\mathrm{C}$ and $\mathrm{B}$ stimuli, and they were as follows: More than C2/C3-B2, More than C2/C1-B2, More than C3/C1-B3, and More than C3/C1-C2, Less than C1/C3-B1, Less than C1/C2-B1, Less than C3/C2-B3, Less than C3/C2-C1 (see Figure 1). The words "Less than" and "More than" represent the arbitrary forms that had been established during pretraining as the contextual cues for Less than and More than, respectively. The first alphanumeric C1, C2, or C3 represents the sample, and the two alphanumerics connected by a hyphen are the comparison stimuli. Choosing the first comparison stimulus was deemed correct (see Figure 1). 


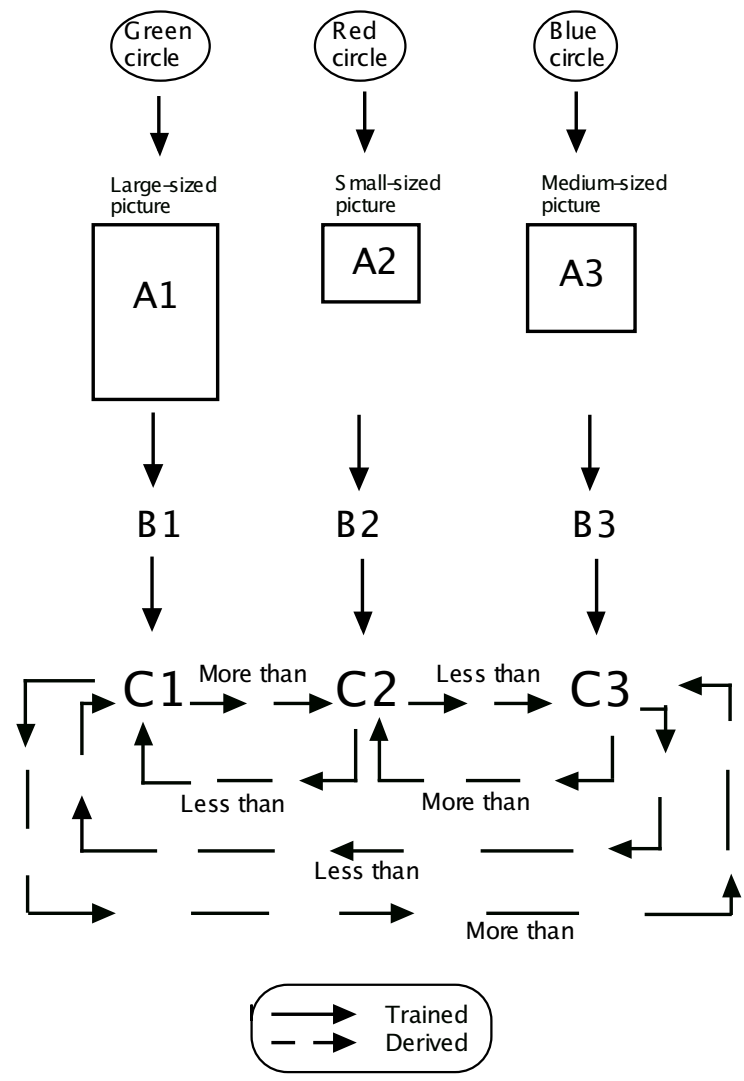

Figure 2. A schematic representation of the sequence of training and testing tasks used in both experiments and the relational network predicted to emerge between the $C$ stimuli during the critical More-than and Less-than probe trials.

Responding in accordance with the predicted relational network required that participants would (a) select C3 and C1 in the presence of the More than cue, given $\mathrm{C} 2$ as the sample, because both $\mathrm{C} 3$ and $\mathrm{C} 1$ participate in derived relations with A3 (medium-sized pictures) and A1 (large-sized pictures), which are of greater physical size than the small-sized pictures indirectly related to $\mathrm{C} 2$; (b) select $\mathrm{C} 1$ in the presence of the More than cue, given $\mathrm{C} 3$ as the sample, because $\mathrm{Cl}$ is indirectly related to Al (large-sized pictures), which is of greater physical size than the medium- and small-sized pictures indirectly related to B3 and C2, respectively; (c) select C3 and C2 in the presence of the Less-than cue, given $\mathrm{C} 1$ as the sample, because both $\mathrm{C} 3$ and C2 participate in derived relations with A3 (medium-sized pictures) and A2 (small-sized pictures), which are of smaller physical size than the large-sized picture indirectly related to $\mathrm{C} 1$; and (d) select $\mathrm{C} 2$ in the presence of the Lessthan cue, given $\mathrm{C} 3$ as the sample, because the $\mathrm{C} 2$ is indirectly related to A2 (small-sized pictures), which is of smaller physical size than the medium- and large-sized pictures indirectly related to B3 and C1, respectively. Figure 2 
gives a schematic representation of the sequence of training and testing tasks from which the derived More-than and Less-than responses were predicted to emerge.

The remainder of the tasks tested for emergent relations between the colored discriminative stimuli and the C stimuli: More than Red-circle/ C3-C2, More than Red-circle/C1-C2, More than Blue-circle/C1-C3, More than Blue-circle/C1-C2, Less than Green-circle/C2-C1, Less than Green-circle/ C3-C1, Less than Blue-circle/C2-C3, and Less than Blue-circle/C2-C1 (see Figure 1). Responding in accordance with the predicted relational network required that participants would (a) select $\mathrm{C} 3$ and $\mathrm{C} 1$ in the presence of the More than cue and given a Red circle as sample, because both C3 and C1 are indirectly related to medium-sized and large-sized pictures, which are of greater physical size than small-sized pictures that followed presentations of Red circles; (b) select $\mathrm{C} 1$ in the presence of the More than cue and given a Blue circle as the sample, because $\mathrm{Cl}$ is indirectly related to Al (large-sized pictures), which is of greater physical size than the medium-size pictures that followed presentations of Blue circles; (c) select C2 and C3 in the presence of the Less than cue and given a Green circle as the sample, because C2 and C3 are indirectly related to small- and medium-sized pictures, which are of smaller physical size than large-size pictures that followed presentations of Green circles; and (d) select C2 in the presence of the Less than cue and given a Blue circle as the sample, because C2 is indirectly related to A2 (small-sized pictures), which is of smaller physical size than medium-size pictures that followed presentations of Blue circles.

\section{Phase 6: Extinction}

This phase was identical to Phase 1, except that sexually explicit stimuli now no longer followed the key-pressing responses. Participants were exposed to this phase once, consisting of one block of 30 trials, 10 of each colored circle, presented quasi-randomly with no more than two consecutive trials of the same type.

\section{Results and Discussion}

Detailed results for each participant across all experimental phases are given in Table 1. Participant 1 required two exposures to discrimination training in order to meet criterion responding. He then passed both the relational pretraining and the test on his first exposure to each. This participant required 9 exposures to the $\mathrm{A}-\mathrm{B}$ and $\mathrm{B}-\mathrm{C}$ relational training in order to meet the responding criterion. Due to a programming error, this participant was not exposed to an equivalence test. On the critical probes, Participant 1 produced near-criterion responding on all of the probes for derived More-than and Less-than relations among the $\mathrm{C}$ and $\mathrm{B}$ stimuli, and between the $\mathrm{C}$ stimuli and the colored discriminative stimuli. That is, he responded correctly on at least four of the five exposures to each of 16 probes, except one. During the extinction phase, he responded consistently and correctly without reinforcement to each of the 30 colored discriminative stimuli presentations.

The remaining two participants were exposed to an equivalence test to ensure that the trained equivalence relations were well established before exposure to the critical More-than and Less-than relational probes. Participant 2 produced 100\% correct responding on her first exposure to the 
Table 1

Total Correct Responses During Phases 1-6

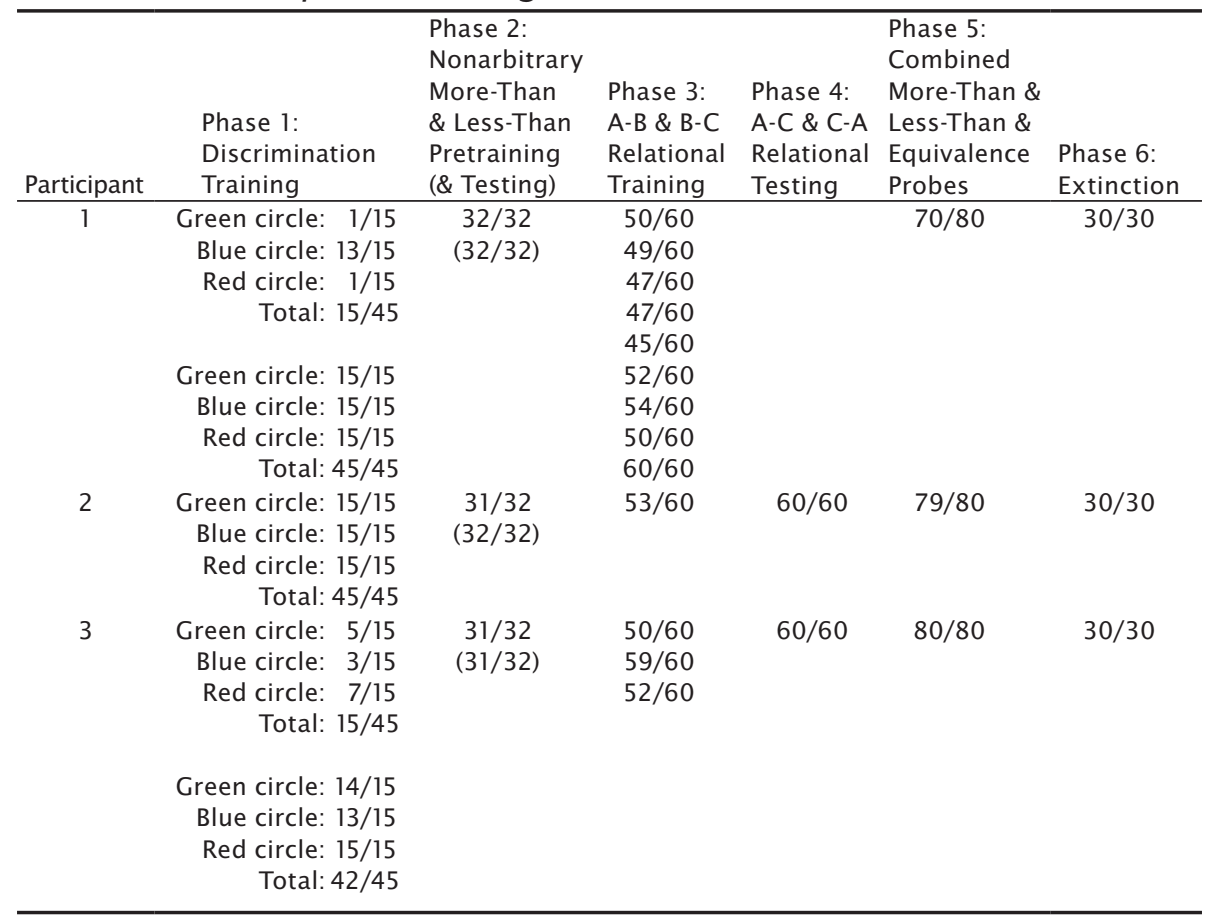

discrimination training phase. She then passed relational pretraining and testing on her first exposure to each. During the relational training phase this participant failed to meet the criterion of 54 out of 60 (i.e., no more than one incorrect response on each of the 6 trial types). Specifically, she produced only 8 correct responses out of 10 to one of the trial types. However, due to experimenter error, she was allowed to proceed to the test phase, which she passed on her first exposure with $100 \%$ correct responding. At exposure to the critical relational test probes, she responded correctly on 79 of the 80 trials. Finally, during the extinction phase, she responded correctly to each of the three colored discriminative stimuli on $100 \%$ of trials.

Participant 3 required two exposures to the discrimination training phase before producing criterion responding. He then passed both relational pretraining and the subsequent test on his first exposure. He then required three exposures to the relational training, after which he passed the relational test on his first exposure. He subsequently produced $100 \%$ correct responding on the More-than and Less-than relational probes. Finally, he responded correctly on all discrimination trials during the extinction phase.

In summary, all three participants derived the predicted Morethan and Less-than relations among the $\mathrm{B}$ and $\mathrm{C}$ stimuli and among the discriminative stimuli and the $\mathrm{C}$ stimuli, illustrating that the nonarbitrary properties of size transformed the arbitrary relational properties of the sexual stimuli. The observed pattern of responding can be explained only in terms of the formal More-than and Less-than relations that obtained 
between the sexual stimuli and the arbitrary equivalence relations in which those stimuli participated. Without these relations coming to bear on the responses of participants during the critical probe phase, participants have no grounds on which to respond, consistently or inconsistently, to any of the arbitrary stimuli as more than or less than each other. In effect, the nonarbitrary properties of the primary sexual stimuli transformed the relational properties of all stimuli in the relational network such that they spontaneously entered into derived More-than and Less-than relations with each other.

It is important to understand that the observed performance may well have emerged even for participants for whom the sexual stimuli were not putatively reinforcing. That is, given the current preparation, it is sufficient that participants respond to the nonarbitrary relations between the sexual stimuli (i.e., More than and Less than) and between the discriminative stimuli and sexual stimuli (i.e., equivalence) for the derivation of a range of novel More-than and Less-than relations. Thus it may well have been the feedback delivered by the computer, rather than the sexual stimuli, that reinforced participants' discriminative behavior toward the colored discriminative stimuli. This observation, however, in no way challenges the importance of the observed transformation effect but merely calls into question the nature of the formal relation between the sexual stimuli employed (i.e., it may have been entirely based on physical size, or it may have been based on the amount of sexual arousal produced by each).

It is interesting that all three participants responded consistently in extinction (Phase 6) to the discriminative stimuli in the absence of sexual images. This finding runs counter to what we might expect of normal contingency-shaped behavior. Such a performance suggests that either the discriminative behavior established at the outset was extremely fluent and resistant to extinction or the discriminative stimuli or the operant response, or both, acquired sexual reinforcing properties. Indeed, this latter possibility should not be surprising, given that it has been well established in previous research that arbitrary stimuli can acquire sexually arousing properties by virtue of their participation in derived relations with primary sexual reinforcers (Roche \& Barnes, 1997; Roche et al., 2000). On the other hand, one of the participants was a self-declared heterosexual female and was unlikely to have been sexually aroused by the stimuli employed.

To address the possibility that the relation between the sexual images was based on their strengths as sexual reinforcers, at least for some participants, a second experiment was conducted. In Experiment 2, participants were exposed to a free-operant procedure intended to establish the effectiveness of the sexual stimuli as reinforcers at the outset. Participants were also exposed to a modified free-operant phase in which the $\mathrm{C}$ stimuli were presented as derived discriminative stimuli.

\section{Experiment 2}

Experiment 1 established that it is possible for the functions of entire networks of arbitrary stimuli to transform in accordance with the nonarbitrary relational properties of stimuli. However, it is unclear 
whether the sexual stimuli employed functioned as sexual reinforcers for some or all of the participants. To further explore this possibility, Experiment 2 involved exposing participants to a free-operant procedure to examine the reinforcing properties of the sexual stimuli at the outset. After the critical probes for derived More than and Less than relations, participants were also exposed to a free-operant phase in which the $C$ stimuli were presented in extinction. Thus Experiment 2 replicated the novel and complex transformation effect observed in Experiment 1, regardless of the sexually reinforcing properties of the sexual stimuli. However, this experiment also allowed us to observe the transformation of functions by the sexually reinforcing properties of stimuli for those participants for whom the sexual images were reinforcing at the outset. Thus we expected to observe an interesting transformation of functions for all participants and to observe the emergence of reinforcing properties for $C$ stimuli only for those participants for whom the sexual images were reinforcing during the initial free-operant stage.

\section{Procedure}

Experiment 2 employed the same procedure as Experiment 1, except for the following important differences. A forced-choice version of the discrimination training procedure was used in Phase 1, and a free-operant phase was used at the outset and the completion of the experiment.

In Experiment 2, Phase 1 consisted of a stimulus pairing procedure in which each colored circle was paired with a sexually explicit stimulus of a different size. Phase 2 involved a free-operant baseline assessment of participants' preferences for the colored circles. Phase 3 involved nonarbitrary training and testing, and Phases 4 and 5 trained A-B and $\mathrm{B}-\mathrm{C}$ conditional discriminations and tested for $\mathrm{A}-\mathrm{C}$ transitive and $\mathrm{C}-\mathrm{A}$ equivalence relations, respectively. In Phase 6 , emergent relations of More than and Less than were tested, and finally, in Phase 7 a freeoperant test was undertaken in which the $C$ stimuli were presented in the absence of feedback.

Phase 1: Stimulus pairing. The aim of this phase was to pair each of the three discriminative stimuli (colored circles) with sexually explicit stimuli of differing sizes in a forced-choice procedure. The instructions for this phase were as follows:

Your task is to look at the image presented in the centre of this computer screen and to "click on" it using the computer's mouse. Please pay attention to what you are clicking on. During this phase you will also see several sexually explicit images. If you have any questions please ask the experimenter now. Press any key to begin.

At the beginning of each stimulus-pairing trial, one of the colored circles (green, red, or blue) was presented in the center of the screen. Clicking on the colored circle produced a sexually explicit picture in one of the three sizes, depending on the color of the circle. Clicking on the Green circle was immediately followed by a brief $0.5 \mathrm{~s}$ high-pitched beep and then $1 \mathrm{~s}$ later by the presentation of a large-sized sexually explicit stimulus that remained on screen for $7 \mathrm{~s}$. Clicking on the Blue circle was 
immediately followed by a brief $0.5 \mathrm{~s}$ high-pitched beep and then $1 \mathrm{~s}$ later by the presentation of a medium-sized sexually explicit stimulus that remained on screen for $7 \mathrm{~s}$. Clicking on the Red circle was immediately followed by a brief $0.5 \mathrm{~s}$ high-pitched beep and then $1 \mathrm{~s}$ later by the presentation of a small-sized sexually explicit stimulus that remained on screen for $7 \mathrm{~s}$. There were no programmed consequences for clicking on any other area of the screen.

Forty-five trials were presented in this phase, 15 trials of each of the colored circles, which were presented quasi-randomly with no more than two consecutive trials of the same type. Participants were exposed to this phase only once.

Phase 2: Free-Operant Baseline. The aim of this phase was to establish a baseline measure of participants' preference for one of the three colored-circle discriminative stimuli. Participants were presented with the following instructions:

Your task is to look at the images presented in the center of this computer screen and to "click on" one of them using the computer mouse. You may press any button you choose. There is no correct choice and no feedback will be provided. During this phase you will also see several sexually explicit images. If you have any questions, please ask the experimenter now.

On every trial, the three colored circles (Green, Blue, Red) were presented simultaneously in the top half of the screen, with positions randomized. Clicking on one of the colored circles with the computer mouse produced a display of a small-, medium-, or large-sized sexually explicit stimulus that remained on screen for $7 \mathrm{~s}$. Clicking on the Greencircle was followed by the presentation of a large-sized sexually explicit stimulus, clicking on the Blue-circle was followed by the presentation of a medium-sized sexually explicit stimulus, and clicking on the Redcircle was followed by the presentation of a small-sized sexually explicit stimulus. This phase ended after 30 trials, and participants were given just one exposure.

Phase 3 (nonarbitrary More-than and Less-than pretraining and testing), Phase 4 (A-B and B-C relational training), Phase 5 (A-C and C-A relational testing), and Phase 6 (probes for combined emergent relations of More than and Less than and equivalence) were identical to Experiment 1.

Phase 7: Free-Operant Probes. The aim of this phase was to test for a transformation of functions by presenting the $C$ stimuli that were related via equivalence with the colored circles. The following instructions were provided:

Your task is to look at the images presented in the centre of this computer screen and to "click on" one of them using the computer mouse. You may press any button you choose. There is no correct choice and no feedback will be provided. During this phase you will not see sexually explicit images immediately after making a choice. Instead, the computer will record your choices and all of the relevant sexual images will be presented automatically in sequence at the end of this phase of the experiment. If you have any questions, please ask the experimenter now. 
Participants were instructed that sexual images would be presented at the end of the phase, yet none was presented. This brief deception was considered necessary to maintain on-task attention and to ensure consistency in responding across the probe trials. On every trial, C1, C2, and C3 were simultaneously presented in the top half of the screen, with positions randomized. Clicking on one of the stimuli removed the display and immediately presented the next trial. Thirty trials were presented, with each of the $\mathrm{C}$ stimuli appearing 10 times, and participants were exposed to this phase only once.

\section{Results and Discussion}

Table 2 shows the detailed results for each participant for all experimental phases except Phase 1. Participant 4 dispersed responses equally among the three colored circles during the free-operant phase (Phase 2), choosing to view an equal number of small, medium, and large sexually explicit images. This participant then needed 10 exposures to the nonarbitrary relational pretraining to reach criterion and passed the nonarbitrary relational test on his first exposure. After three exposures to Phase 3 (A-B and B-C training), a break of one day occurred between sessions. On his return to the laboratory, Participant 4 was reexposed to the free-operant phase and once again dispersed responses across the three Green, Red, and Blue discriminative stimuli (S $S^{\mathrm{D}}$ 9, 11, 10, respectively). He then went on to pass the relational pretraining and testing on the first exposure, after which he required five exposures to the relational training to meet criterion. This participant then reached the consistency criterion on the second exposure to the More-than and Less-than probes (Phase 6). On the final free-operant phase, this participant responded by clicking on the $\mathrm{Cl}$ stimulus, as predicted on all trials. That is, given the three $\mathrm{C}$ stimuli as $\mathrm{S}^{\mathrm{D}} \mathrm{S}(\mathrm{C} 1, \mathrm{C} 2, \mathrm{C} 3)$, the participant responded to the $\mathrm{C} 1$ stimulus on every trial, as this was in a derived equivalence relation with the Green discriminative stimulus that had been paired with large-sized sexually explicit images.

Participant 5 also dispersed responses among the Green, Blue and Red $S^{\mathrm{D}} \mathrm{S}(12,7,11)$ respectively, during the initial free-operant phase. He then went on to pass both the relational pretraining and testing phases on his first exposure. On his second exposure to the relational training phase, he produced criterion responding and subsequently passed the relational test on his first attempt. This participant failed to derive the predicted Morethan and Less-than relations within three exposures to the probe phase, and his first experimental session was terminated. On a subsequent day he once again dispersed responses among the Green, Blue, and Red $S^{\text {D }} S$ $(14,9,7$, respectively) during the initial free-operant phase. He passed the relational pretraining and testing and the relational training and testing on his first exposures. However, he once again failed to derive the predicted More-than and Less-than relations on his first exposure. Due to time constraints on the part of the participant, the session was terminated. As we would expect, given his failure to derive the predicted relations, he also dispersed his responses among the C1, C2, and C3 stimuli (10, 10, 10, respectively) during the final free-operant phase. 
Table 2

Total Correct Responses During Phases 2-7

\begin{tabular}{|c|c|c|c|c|c|c|}
\hline Participant & $\begin{array}{l}\text { Phase 2: } \\
\text { Free-Operant } \\
\text { Baseline }\end{array}$ & $\begin{array}{l}\text { Phase } 3: \\
\text { Nonarbitrary } \\
\text { More-Than } \\
\text { \& Less-Than } \\
\text { Pretraining } \\
\text { (\& Testing) }\end{array}$ & $\begin{array}{l}\text { Phase 4: } \\
\text { A-B \& B-C } \\
\text { Relational } \\
\text { Training }\end{array}$ & $\begin{array}{l}\text { Phase 5: } \\
\text { A-C \& C-A } \\
\text { Relational } \\
\text { Testing }\end{array}$ & $\begin{array}{l}\text { Phase 6: } \\
\text { Combined } \\
\text { More-Than \& } \\
\text { Less-Than } \\
\text { \& Equivalence } \\
\text { Probes }\end{array}$ & $\begin{array}{l}\text { Phase 7: } \\
\text { Free- } \\
\text { Operant } \\
\text { Probes }\end{array}$ \\
\hline \multirow[t]{17}{*}{4} & Green circle: 10 & $13 / 32$ & $34 / 60$ & & & \\
\hline & Blue circle: 10 & $15 / 32$ & $34 / 60$ & & & \\
\hline & Red circle: 10 & $15 / 32$ & $32 / 60$ & & & \\
\hline & & $12 / 32$ & & & & \\
\hline & & $9 / 32$ & & & & \\
\hline & & $13 / 32$ & & & & \\
\hline & & $19 / 32$ & & & & \\
\hline & & $13 / 32$ & & & & \\
\hline & & $28 / 32$ & & & & \\
\hline & & $31 / 32$ & & & & \\
\hline & & $(32 / 32)$ & & & & \\
\hline & Reexposure & & & & & \\
\hline & Green circle: 9 & $32 / 32$ & $38 / 60$ & $60 / 60$ & $75 / 80$ & $\mathrm{Cl}: 30$ \\
\hline & Blue circle: 11 & $(30 / 32)$ & $26 / 60$ & & $80 / 80$ & C2: 0 \\
\hline & Red circle: 10 & & $28 / 60$ & & & C3: 0 \\
\hline & & & $43 / 60$ & & & \\
\hline & & & $59 / 60$ & & & \\
\hline \multirow[t]{7}{*}{5} & Green circle: 12 & $30 / 32$ & $53 / 60$ & $60 / 60$ & $32 / 80$ & \\
\hline & Blue circle: 7 & $(32 / 32)$ & $60 / 60$ & & $24 / 80$ & \\
\hline & Red circle: 11 & & & & $22 / 80$ & \\
\hline & Reexposure & & & & & \\
\hline & Green circle: 14 & $32 / 32$ & $59 / 60$ & $60 / 60$ & $20 / 80$ & $\mathrm{C} 1: 10$ \\
\hline & Blue circle: 9 & $(32 / 32)$ & & & & C2: 10 \\
\hline & Red circle: 7 & & & & & C3: 10 \\
\hline \multirow[t]{6}{*}{6} & Green circle: 28 & $31 / 32$ & $49 / 60$ & $59 / 60$ & $48 / 80$ & \\
\hline & $\begin{array}{l}\text { Blue circle: } 1 \\
\text { Red circle: } 1\end{array}$ & $(32 / 32)$ & $59 / 60$ & & $37 / 80$ & \\
\hline & Re-exposure & & & & & \\
\hline & Green circle: 30 & $32 / 32$ & $60 / 60$ & $59 / 60$ & $78 / 80$ & $\mathrm{C} 1: 30$ \\
\hline & Blue circle: 0 & $(32 / 32)$ & & & & C2: 0 \\
\hline & Red circle: 0 & & & & & C3: 0 \\
\hline \multirow[t]{6}{*}{7} & Green circle: 11 & $32 / 32$ & $53 / 60$ & $59 / 60$ & $1 / 80$ & \\
\hline & $\begin{array}{l}\text { Blue circle: } 9 \\
\text { Red circle: } 10\end{array}$ & $(32 / 32)$ & $60 / 60$ & & $11 / 80$ & \\
\hline & & & Reexposure & & & \\
\hline & & & $60 / 60$ & $60 / 60$ & $4 / 80$ & $\mathrm{C} 1: 8$ \\
\hline & & & & & & C2: 14 \\
\hline & & & & & & C3: 8 \\
\hline \multirow[t]{3}{*}{8} & Green circle: 10 & $32 / 32$ & $53 / 60$ & $60 / 60$ & $80 / 80$ & $\mathrm{C} 1: 9$ \\
\hline & Blue circle: 14 & $(32 / 32)$ & $60 / 60$ & & & $C 2: 6$ \\
\hline & Red circle: 6 & & & & & C3: 15 \\
\hline \multirow[t]{3}{*}{9} & Green circle: 30 & $32 / 32$ & $59 / 60$ & $60 / 60$ & $80 / 80$ & $\mathrm{C} 1: 30$ \\
\hline & Blue circle: 0 & $(32 / 32)$ & & & & C2: 0 \\
\hline & Red circle:0 & & & & & C3: 0 \\
\hline
\end{tabular}

Participant 6 showed a clear preference for the Green $S^{\mathrm{D}}$, associated with the presentation of large sexual stimuli, by responding to it on 28 of the 30 free-operant trials. He then passed relational pretraining and testing on his first attempt before requiring two exposures to the relational training 
phase prior to passing, and then proceeded to pass the relational test on his first exposure. He failed, however, to derive the predicted More-than and Less-than relations and so was reexposed to the free-operant and subsequent phases on the following day. Specifically, he responded exclusively to the Green $S^{D}$ during the initial free-operant phase, after which he passed each of the nonarbitrary relational pretraining and testing and relational training and testing phases on his first attempts. Upon reexposure to the critical probe phase, he produced criterion responding on his first attempt. This participant also showed a clear preference for the $\mathrm{C} 1$ stimulus during the final free-operant phase. That is, he responded to $\mathrm{C} 1$ on each of the 30 trials presented in extinction.

Participant 7 dispersed responses among the three colored $\mathrm{S}^{\mathrm{D}} \mathrm{s}$ during the initial free-operant phase. He then passed relational pretraining and testing on his first exposure. He required two exposures to relational training before reaching criterion responding and then passed the relational test on the first exposure. He failed, however, to produce the predicted More-than and Lessthan relations within two exposures and was asked to return to the laboratory on the following day. During the second session he was exposed only to the relational training and testing phases, which he passed on the first attempts. However, he once again failed to produce the predicted derived relations during the probe phase, and his performance was sufficiently erratic that he was moved directly to the final free-operant phase before his participation was terminated. In the final phase, he dispersed his responses among the C1, C2, and C3 stimuli, as we would expect for a participant who had not produced the required derived relations.

Participant 8 showed a preference for the Blue $S^{\mathrm{D}}$ during the free-operant phase by selecting it on 14 trials, the Green $S^{D}$ on 10 trials, and the Red $S^{D}$ on 6 trials. He reached criterion on his first exposure to the nonarbitrary relational pretraining and testing phase before requiring two exposures to the relational training phase and then immediately passing the relational test. This participant then produced the predicted derived performance on his first exposure to the More/Less probe phase. During the final free-operant phase, he selected the C2 stimulus on 15 trials, C1 on 9 trials, and C3 on 6 trials. This derived preference for $\mathrm{C} 2$ was consistent with this participant's preference for the medium-sized sexually explicit stimulus observed in Phase 2 that participated in a derived equivalence relation with $\mathrm{C} 2$.

Participant 9 showed a preference for the Green $S^{D}$ during the initial freeoperant phase by responding to it on all trials. He then passed the nonarbitrary relational pretraining and testing and relational training and testing on his first exposure. Participant 9 then produced the predicted More-than and Less-than derived relations on his first exposure to the critical probe phase and subsequently responded to the $\mathrm{C} 1$ stimulus on all trials during the final free-operant phase.

In summary, of the six participants who participated in Experiment 2, four demonstrated the predicted derived More-than and Less-than relations. Interestingly, of these four participants, two (S6 and S9) demonstrated a performance during the initial free-operant phase which suggested that the large sexual images were more reinforcing than the medium or small sexual images (i.e., they responded almost exclusively to the Green $S^{\mathrm{D})}$ ). Moreover, as predicted, these and only these two participants responded exclusively to the arbitrary $\mathrm{C} 1$ stimulus (i.e., related through equivalence to the Green $\mathrm{S}^{\mathrm{D}}$ ) 
during the final free-operant phase presented in extinction. In contrast, the remaining participants dispersed responses among the discriminative stimuli during the initial free-operant stage and, as expected, also dispersed responses to the $\mathrm{C} 1, \mathrm{C} 2$, and $\mathrm{C} 3$ stimuli during the final free-operant stage. This finding strongly suggests that for two participants, the $\mathrm{C}$ stimuli functioned as derived $\mathrm{S}^{\mathrm{D}} \mathrm{s}$ for sexual reinforcement.

\section{General Discussion}

Experiment 1 demonstrated a transformation of functions in accordance with the nonarbitrary relational properties of sexual stimuli. In the critical probes phase, a large number of derived More-than and Less-than relations emerged among the $\mathrm{B}$ and $\mathrm{C}$ stimuli and among the discriminative stimuli and the $\mathrm{C}$ stimuli. Experiment 2 used a modified free-operant procedure to establish the effectiveness of the sexual stimuli as putative reinforcers at the outset. The findings suggest that the functions of the $\mathrm{C}$ stimuli were transformed in accordance with the nonarbitrary relational properties of sexual reinforcers for at least two participants.

These data extend previous research (Dymond \& Barnes, 1995, 1996; Roche \& Barnes, 1997; Whelan et al., 2006) by demonstrating a transformation of functions in accordance with the comparative relational frame of More than and Less than. However, there are a number of important differences between the current procedures and those used in previous transformation research. Typically, a discrete, nonrelational function is first established for one member of a derived relation before transformation is then tested with presentations of the remaining relational stimuli, in the absence of further training (Dymond \& Rehfeldt, 2000, p. 241). In the current study, however, the nonarbitrary relational properties of size were not directly established for any members of the relational network. Instead, the formal nonarbitrary and naturally occurring properties of the A1, A2, and A3 stimuli participated in the trained relational network. Also, the More-than and Less-than relations that transformed the functions of the $\mathrm{C}$ stimuli were not directly established by the experimenters. Indeed, no More-than or Less-than relations were trained at any stage, other than in establishing contextual functions for two cues. Nevertheless, after the equivalence training and testing procedure, a series of More-than and Less-than relations spontaneously emerged under contextual control between the $\mathrm{C}$ stimuli and between the colored circles. This finding extends previous derived transformation research insofar as it demonstrates how a limited number of trained relations of one type can produce a massive transformation of functions across an entire relational network involving multiple stimulus relations.

It is likely that the use of the free-operant phase employed at the outset of Experiment 2 established the sexual stimuli as putative reinforcers for two participants. Furthermore, these participants (S6 and S9) responded to the C stimuli in a free-operant context as if they were discriminative for sexual reinforcement. That is, these participants responded exclusively to the $\mathrm{C} 1$ stimulus as predicted by a relational account. It would appear that this performance can be predicted only by appealing to the arbitrary application of the nonarbitrary relational properties of the sexual reinforcers to the $C$ stimuli.

It could be argued that in the absence of control by the reinforcing properties of the sexual stimuli, all participants may simply have distributed 
their responses randomly or stereotypically during the free-operant phases. In other words, natural variance across participants' response patterns to the $C$ stimuli during the final free-operant stage may explain the lack of similarity in performances. Such an account, however, would need to address the predicted absence of stereotypical response patterns during the final free-operant stage for participants other than S6 and S9. Specifically, S6 and S9 responded consistently to the Green $S^{\mathrm{D}}$ as predicted, during the initial free-operant stage, and to $\mathrm{C} 1$, as predicted, during the final free-operant stage. It would appear to be more than coincidence that the predicted $100 \%$ correspondence across the initial and final free-operant stages was observed for these participants. Moreover, the remaining participants' performances also corresponded across the free-operant stages, as predicted. That is, the distribution of responses to the colored $S^{\mathrm{D}} S$ at the outset was reliably predictive of a distribution of responses to the $\mathrm{C}$ stimuli during the final free-operant stage. In addition, an account based on an assumption of random variation in responding across participants during the final freeoperant stage would also need to assume that the sexual images were not reinforcing for any of the participants. This is highly unlikely, given the origin of the images in mainstream British pornographic publications. In any case, the most important contribution of the experiments is not to demonstrate that the formal properties of sexual reinforcing stimuli can transform the functions of an entire network of multiple stimulus relations for all or most participants, but to demonstrate the formal properties of any stimuli can produce this effect.

For researchers interested specifically in the unique susceptibility of stimuli to transformations of function by sexual reinforcers, we suggest that an explicit stimulus matching procedure be employed to assess the sexual categorization of stimuli at the outset (see Roche \& Barnes, 1996). Furthermore, rating scales and verbal reports may be used to determine participants' sexual feelings toward the sexual images. Finally, a form of protocol analysis or "talk aloud" procedure (e.g., Cabello \& O'Hora, 2002; Hayes, 1986; Rehfeldt \& Dixon, 2000) might be used to identify not only the sexual responses of participants toward the stimuli but also any verbal rules they may be using in making response choices during the critical phases of the experiment. Despite some ambiguity in the precise functions of the sexual images employed in the current study, it nevertheless remains the case that the transformation effects caused by the participation of the sexual stimuli in the relational network was well controlled and replicated across two experiments.

Both Experiments 1 and 2 employed a two-choice comparison procedure during all relational training and testing phases. Such procedures have often been criticized as allowing for the possibility that participants' selections may be influenced by extraneous sources of stimulus control (Carrigan \& Sidman, 1992; but see Boelens, 2002). However, in research on More-than and Less-than relations it is necessary to present two comparisons for the following reason. Presenting only two comparisons avoids the ambiguous situation in which, for example, given the More-than cue with three stars as the sample and two, four, and six stars as the comparisons, there would be two correct choices (i.e., both the four stars and six stars are more than the three-star sample). Indeed, a three-comparison-type task, may inadvertently establish the More-than cue as functionally equivalent to an 
Opposite cue. For instance, a participant might consistently select the sixstar comparison in the presence of the three-star sample and therefore fail to learn that the "more-than" four-star comparison is also correct (i.e., the More-than cue would control selection of the opposite comparison, rather than a comparison that was simply more than the sample). The use of only two comparisons thereby ensured that Opposite relational control could not occur during More-than and Less-than pretraining and in the critical probes phase (Dymond \& Barnes, 1995; O’Hora et al., 2002).

To the behavior analytic sex researcher, the analysis of stimulus control over sexual responses provides an important insight into the etiology and maintenance of both normal and deviant sexual behavior. To date, a range of behavioral sex research studies concerned with stimulus control have examined habituation to sexual stimuli (e.g., Koukounas \& Over, 1993; Meuwissen \& Over, 1990; O’Donohue \& Plaud, 1994; Plaud, Gaither, Henderson, \& Devitt, 1997), the respondent conditioning of sexual arousal to arbitrary stimuli (e.g., Langevin \& Martin, 1975; Plaud \& Martini, 1999; Rachman \& Hodgson, 1968; Roche \& Barnes, 1997), and the operant conditioning of sexual arousal (e.g., Rosen, Shapiro, \& Schwartz, 1975; Schaefer \& Colgan, 1977). As a result, a modest but relatively fruitful laboratory-based learning approach to sexual behavior has been developed. However, the relevance of the current findings to real-world examples of sexual behavior is not immediately obvious. More specifically, it is difficult to provide an example of the emergence of sexual reinforcing functions through the process explored here, in the absence of a concurrent transformation of sexual eliciting functions and the direct association of arbitrary stimuli with sexual reinforcement. This problem is illustrated by the following example.

Imagine a scenario where within the sexual culture of a bar, buying a person a glass of champagne signaled the desire for full sexual intercourse, buying a glass of wine signaled a desire for heavy petting and perhaps oral sex, and buying a glass of beer signaled the desire for flirtatious conversation. In this example, patrons of the bar may come to derive relations of More than and Less than between the various drinks, whereby champagne is more than wine and wine is more than beer. Under these conditions champagne may even acquire more sexual reinforcing properties than beer such that thinking of champagne may produce mild covert feelings of sexual arousal. However, while this process may occur in principle, more powerful and less subtle processes of respondent and operant conditioning will quickly swamp it. That is, after several occasions on which buying champagne and drinking it leads to full sexual intercourse, the champagne acquires sexual arousing properties by virtue of simple respondent processes (i.e., the champagne becomes a conditioned stimulus for subsequent sexual pleasure) and traditional operant processes (i.e., the champagne becomes an $\mathrm{S}^{\mathrm{D}}$ for the availability of sexual reinforcement after sexual advances). The champagne may also function as an establishing operation for responses to discriminative stimuli produced by the patron of the bar, such as flirtatious verbal signals or modes of dress or bodily posture. Thus, while the derived relations between the various drinks may have emerged spontaneously to begin with, their potency as derived sexual reinforcers rapidly becomes insignificant compared with their potency as directly established respondent or discriminative stimuli for 
the availability of sexual reinforcement. In summary, derived relational responding will often come under direct contingency control in the world outside the laboratory, and its origins in relational processes will often become obscured over time.

While the processes demonstrated in this study may not easily map directly onto discrete examples of the acquisition of sexual arousal functions for arbitrary stimuli, the role of verbal process in human sexual behavior is not negated. The demonstration of this process makes two important contributions to the behavioral sex research agenda. First, it demonstrates in principle that sexual stimuli can participate in verbal networks in the real world, and it is therefore possible that this may enhance the sexual functions of the relevant stimuli, however slightly. Second, the spontaneous emergence of large complex relational networks containing sexual stimuli makes possible the emergence of further derived relations that may in turn facilitate a further derived transformation of functions. For instance, imagine a preparation like the one used in the current study in which responses to the arbitrary stimuli $\mathrm{A}, \mathrm{B}$, and $\mathrm{C}$ lead to the delivery of small, medium, and large sexual reinforcers, respectively. Now imagine that equivalence relations are established between $\mathrm{A}, \mathrm{B}$, and $\mathrm{C}$ and the new stimuli $\mathrm{D}, \mathrm{E}$, and F, respectively. We would now expect $\mathrm{F}$ to produce the highest level of responding and to function as the most potent sexual reinforcer owing to its relation to $C$. In this example, the relational properties of $\mathrm{A}, \mathrm{B}$, and $\mathrm{C}$ will continue to spread outward to all stimuli added to the network (i.e., D, E, and F). Thus the current analysis identifies a way in which natural language processes may help to produce novel forms of sexual behavior in the real world that would require more than a simple account in terms of the transfer of discrete nonrelational functions through derived relations.

It remains possible that the effects observed in the current study would have been obtained with any stimuli of varying size, and the effect may reflect only a form of complex derived relational responding toward the sexual images and related stimuli. Thus, it could be argued, we cannot know for certain how the $\mathrm{C}$ stimuli in the current study would have functioned as sexual reinforcers in the world outside the laboratory. Nevertheless, the fact that such a complex pattern of derived responding in accordance with More-than and Less-than relations emerged among the stimuli employed suggests that at least some of the functions of sexual stimuli in the natural world, including their reinforcing functions, can and likely do transform in accordance with similar relational networks. Even more important, the current study demonstrated stimulus control over highly complex relational responding, including relational responding under the control of contextual cues that were never employed during the relational training phase. Thus the current findings supplement the literature on derived relations in general by showing the emergence of derived relational responding under novel forms of contextual control established only by contact with the formal features of a series of putative sexual reinforcers. To this extent these findings provide at least a starting point for researchers interested in applying the analysis of derived relations to the understanding of real-world naturalistic behavioral phenomena such as the acquisition of sexual stimulus functions and sexual preference more generally. 


\section{References}

BARNES, D. (1994). Stimulus equivalence and relational frame theory. The Psychological Record, 44, 91-124.

BARNES, D., \& KEENAN, M. (1993). A transfer of functions through derived arbitrary and non-arbitrary stimulus relations. Journal of the Experimental Analysis of Behavior, 59, 61-81.

BARNES-HOLMES, D., HAYES, S. C., DYMOND, S., \& O'HORA, D. (2001). Multiple stimulus relations and the transformation of stimulus functions. In Hayes, S. C., Barnes-Holmes, D., \& Roche, B. (Eds.), Relational frame theory: A post-Skinnerian account of human language and cognition (pp. 51-71). New York: Kluwer Academic/Plenum.

BOELENS, H. (2002). Studying stimulus equivalence: Defense of the twochoice procedure. The Psychological Record, 52, 305-314.

CABELLO, F., \& O'HORA, D. (2002). Addressing the limitations of protocol analysis in the study of complex human behaviour. International Journal of Psychology and Psychological Therapy, 2, 115-130.

CARRIGAN, P. F., \& SIDMAN, M. (1992). Conditional discrimination and equivalence relations: A theoretical analysis of control by negative stimuli. Journal of the Experimental Analysis of Behavior, 58, 183-204.

COHEN, J. D., MACWHINNEY, B., FLATT, M., \& PROVOST, J. (1993). Psyscope: A new graphic interactive environment for designing psychology experiments. Behavioral Research Methods, Instruments and Computers, $25,257-271$.

DOUGHER, M. J., AUGUSTON, E., MARKHAM, GREENWAY M. R., \& WULFERT, E. (1994). The transfer of respondent eliciting and extinction functions through stimulus equivalence classes. Journal of the Experimental Analysis of Behavior, 62, 331-351.

DOUGHER, M., PERKINS, D. R., GREENWAY, D., KOONS, A., \& CHIASSON, C. (2002). Contextual control of equivalence-based transformation of functions. Journal of the Experimental Analysis of Behavior, 78, 63-94.

DYMOND, S., \& BARNES, D. (1995). A transformation of self-discrimination response functions in accordance with the arbitrarily applicable relations of sameness, more than, and less than. Journal of the Experimental Analysis of Behavior, 64, 163-184.

DYMOND, S., \& BARNES, D. (1996). A transformation of self-discrimination response functions in accordance with the arbitrarily applicable relations of sameness and opposition. The Psychological Record, 46, 271-300.

DYMOND, S., \& REHFELDT, R. (2000). Understanding complex behavior: The transformation of stimulus functions. The Behavior Analyst, 23, 239-254.

FIELDS, L., ADAMS, B. J., VERHAVE, T., \& NEWMAN, S. (1990). The effects of nodality on the formation of equivalence classes. Journal of the Experimental Analysis of Behavior, 53, 345-358.

HAYES, S. C. (1986). The case of the silent dog: Verbal reports and the analysis of rules. A review of K. Anders Ericsson and Herbert A. Simon, "Protocol analysis: Verbal reports as data." Journal of the Experimental Analysis of Behavior, 45, 351-363.

HAYES, S. C. (1994). Relational frame theory: A functional approach to verbal events. In S. C. Hayes, L. J. Hayes, M. Sato, \& K. Ono (Eds.), Behavior analysis of cognition and language (pp. 9-30). Reno, NV: Context Press. 
HAYES, S. C., BARNES-HOLMES, D., \& ROCHE, B. (2001). Relational frame theory: A post-Skinnerian account of human language and cognition. New York: Kluwer Academic/Plenum Press.

KOUKOUNAS, E., \& OVER, R. (1993). Habituation and dishabituation of male sexual arousal. Behaviour Research and Therapy, 31, 575-585.

LANGEVIN, R., \& MARTIN, M. (1975). Can erotic responses be classically conditioned? Behavior Therapy, 6, 350-355.

MEUWISSEN, I., \& OVER, R. (1990). Habituation and dishabituation of female sexual arousal. Behaviour Research \& Therapy, 28, 217-226.

O'DONOHUE, W., \& PLAUD, J. (1994). The conditioning of human sexual arousal. Archives of Sexual Behavior, 23, 321-344.

O'HORA, D., BARNES-HOLMES, D., ROCHE, B., \& SMEETS, P. M. (2004). Derived relational networks and control by novel instructions: A possible model of generative verbal responding. The Psychological Record, 54, 437-460.

O'HORA, D., ROCHE, B., BARNES-HOLMES, D., \& SMEETS, P. (2002). Response latencies to multiple derived stimulus relations: Testing two predictions of relational frame theory. The Psychological Record, 52, 51-75.

PLAUD, J. J., GAITHER, G. A., HENDERSON, S. A., \& DEVITT, M. K. (1997). The long-term habituation of sexual arousal in human males: A crossover design. The Psychological Record, 47, 385-398.

PLAUD, J. J., \& MARTINI, J. R. (1999). The respondent conditioning of male sexual arousal. Behavior Modification, 23, 254-268.

RACHMAN, S., \& HODGSON, R. J. (1968). Experimentally-induced "sexual fetishism": Replication and development. The Psychological Record, 18, 25-27.

REHFELDT, R. A., \& DIXON, M. R. (2000) Investigating the relation between self-talk and emergent stimulus relations. Experimental Analysis of Human Behavior Bulletin, 18, 28-29.

REHFELDT, R.A., \& HAYES, L. J. (1998). Untrained temporal differentiation and equivalence class formation. The Psychological Record, 48, 481-509.

ROCHE, B., \& BARNES, D. (1996). Arbitrarily applicable relational responding and sexual categorization: A critical test of the derived difference relation. The Psychological Record, 46, 451-475.

ROCHE, B., \& BARNES, D. (1997). A transformation of respondently conditioned functions in accordance with arbitrarily applicable relations. Journal of the Experimental Analysis of Behavior, 67, 275-301.

ROCHE, B., AND BARNES, D. (1998). The experimental analysis of human sexual arousal: Some recent developments. The Behavior Analyst, 21, 37-52.

ROCHE, B., BARNES-HOLMES, D., SMEETS, P. M., BARNES-HOLMES, Y., \& MCGEADY, S. (2000). Contextual control over the derived transformation of discriminative and sexual arousal functions. The Psychological Record, 50, 267-291.

ROCHE, B., STEWART, I., \& BARNES-HOLMES, D. (1999). PsyScope: An easyto-use graphical system for designing and controlling equivalence experiments. Experimental Analysis of Human Behavior Bulletin, 17, 5-7.

ROSEN, R. C., SHAPIRO, D., \& SCHWARTZ, G. E. (1975). Voluntary control of penile tumescence. Psychosomatic Medicine, 37, 479-483.

SCHAEFER, H. H., \& COLGAN, A. H. (1977). Effect of pornography on penile tumescence as a function of reinforcement and novelty. Behavior Therapy, 8, 938-946. 
SIDMAN, M. (1986). Functional analysis of emergent verbal classes, in T. Thompson and M. E. Zeiler (Eds.), Analysis and integration of behavioral units (pp. 213-245). Hillsdale, NJ: Laurence Erlbaum Associates.

SMEETS, P. M., \& BARNES-HOLMES, D. (2003). Children's emergent preferences for soft drinks: Stimulus equivalence and transfer. Journal of Economic Psychology, 24, 603-618.

STEELE, D., \& HAYES, S. C. (1991). Stimulus equivalence and arbitrarily applicable relational responding. Journal of the Experimental Analysis of Behavior, 56, 519-555.

WHELAN, R., \& BARNES-HOLMES, D. (2004). The transformation of consequential functions in accordance with the relational frames of same and opposite. Journal of the Experimental Analysis of Behavior, 82, 177-195.

WHELAN, R., BARNES-HOLMES, D., \& DYMOND, S. (2006). The transformation of consequential functions in accordance with the relational frames of more-than and less-than. Journal of the Experimental Analysis of Behavior, 86, 317-335. 